\title{
ESTUDIO TEÓRICO - EXPERIMENTAL DE VIGAS DE SECCIÓN TRANSVERSAL COMPUESTA, FABRICADAS CON MADERA Y CONTRACHAPADO ESTRUCTURAL (*)
}

\author{
VICENTE PEREZ GALAZ (**)
}

(*) Proyecto FONDECYT N 1950295 - 1995

(**) Ingeniero Civil, U. de Chile, Profesor Asociado, Departamento de Ingeniería en Obras Civiles, Facultad de Ingeniería, Universidad de Santiago de Chile, Avda. B. O'Higgins 3363, Santiago de Chile.

\section{RESUMEN}

El trabajo que se presenta entrega información para el diseño estructural de vigas de sección transversal compuesta tipo Doble - te y Cajón, elementos comunes en la construcción en madera de países desarrollados.

Entrega un proceso de cálculo que considera la normativa chilena y una metodología extraída de la bibliografía australiana, utilizando las características físicas y mecánicas de los materiales nacionales utilizados.

Para verificar el proceso de diseño se planificó una serie de ensayos de flexión en vigas compuestas, a escala real, fabricadas con alas de Pino radiata (Pinus radiata D. DON) y con alma de contrachapado de Coigüe (Nothofagus dombeyi Mirb.,Blume) fabricado en Chile.

La teoría y la experimentación aplicada a los elementos estructurales escogidos dieron diferencias entre sí, resultando que un $66,7 \%$ de las propiedades resistentes experimentales fueron menores que las teóricas entregadas por el método de diseño propuesto.

Finalmente, el estudio propone un ajuste al proceso de diseño teórico, para concretar la concordancia que debe existir entre la experimentación y la teoría.

Palabras Clave: Vigas de Madera, Contrachapado Estructural, Ensayos de Flexión.

\section{ABSTRACT}

This work deals with the design of I and box sections composite timber beams. This type of elements is commonly used in timber constructions in industrialized countries.

The design procedure given here is related to Chilean wood species taking into account both Chilean standards and an Australian design method.

The proposed design method is verified through an experimental study which includes testing of full scale composite timber beams constructed with Radiata D.Don pine flanges and Coigüe (Nothofagus dombeyi Mirb.,Blume) web.

Some differences were found between experimental and theoretical values. The loading capacity experimentally obtained was lower than the theoretically predicted values for $66,7 \%$ of the test specimens. 
Finally, this study proposes a correction to the design procedure in order to obtain a better correlation between theory and experimentation.

Keywords: Wood Beam, Structural Plywood, Flexion Test. 


\section{INTRODUCCIÓN}

Las vigas compuestas de contrachapado y madera son livianas, simples de fabricar, dimensionalmente estables $\mathrm{y}$, con un buen diseño, estructuralmente eficientes y económicas. Además, ellas pueden fabricarse con una contraflecha para controlar las deformaciones.

Las vigas con contrachapado pueden ser usadas en una amplia gama de aplicaciones tales como vigas de techumbre, costaneras, vigas de piso, dinteles, vigas de soporte de moldajes para hormigón, etc.

El objetivo de este estudio es proveer de suficiente información y datos para su diseño estructural de acuerdo a la normativa chilena y a las experiencias australianas.

Se estudian vigas con sección transversal doble-te y cajón. Ver Figura 1. Ellas constan de alas, almas y, normalmente de rigidizadores. Estos ayudan a controlar el volcamiento del alma de la viga y proveer de adecuadas superficies para las uniones de los trozos de contrachapado usados.

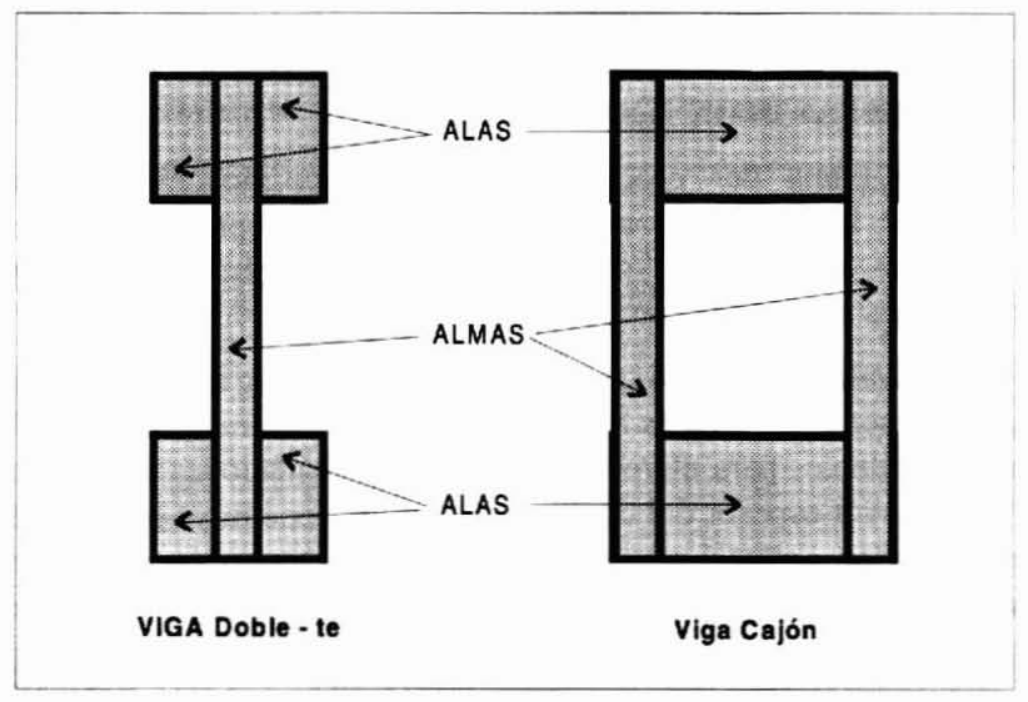

Figura 1. SECCIÓN TRANSVERSAL DE LAS VIGAS ESTUDIADAS.

Es una buena práctica ubicar rigidizadores en aquellos puntos donde existen cargas concentradas para absorber volcamientos localizados en el alma. 
Las alas y las almas pueden ser unidas entre sí mediante un clavado, encolado o clavado - encolado.

Para las vigas encoladas se requiere de un proceso de control de calidad y de correctas prácticas de encolado para asegurar su resistencia y durabilidad durante su vida útil. Esto se obtiene sólo en fábricas, por lo tanto, en general, se recomienda el uso de vigas clavadas. El advenimiento del martillo neumático ha hecho posible una económica fabricación "in situ" de las vigas clavadas. Las vigas encoladas son, obviamente más rígidas pero, la experiencia indica que el diseño en la mayoría de las vigas es gobernado por la resistencia, aún en las clavadas.

La utilización de la combinación de madera de Pino radiata en las alas y contrachapado de Coigüe en las almas, se debe a que ambos tipos de madera se encuentran abundantemente en nuestro país.

El presente trabajo corresponde a la etapa final del Proyecto FONDECYT $\mathbf{N}^{\circ} 1950295$ "Estudio Teórico y Experimental de Vigas de Madera y Contrachapado, de Sección Transversal Compuesta". Antes, se han publicado: " El Contrachapado Estructural" (1) y "Propiedades Físicas y Mecánicas de un Contrachapado de Coigüe, Fabricado en Chile” (2)

\section{SELECCIÓN DE LAS DIMENSIONES DE LA VIGA}

La primera etapa del proceso iterativo de diseño, es seleccionar las dimensiones de la viga y de sus componentes. Esta primera selección está basada en la experiencia o intuición. Sin embargo, las siguientes recomendaciones pueden ayudar.

La razón luz/altura de las vigas cajón puede variar de, aproximadamente, 5:1 para vigas altamente solicitadas hasta 25:1, para costaneras. Las vigas de mayor altura, recomendables para elementos con altas solicitaciones de cizalle (corte), necesitan un mayor espesor del contrachapado que constituye el alma. Las vigas doble-te, a usar en aplicaciones domésticas, pueden tener una razón luz/altura de, aproximadamente, 10:1, mientras que las vigas con poca carga dicha razón pueden ser de alrededor de 18:1. Tal es el caso de las costaneras.

La razón altura/ancho de las vigas, varía entre 3:1 a 15:1, sin embargo la mayoría de la población cae entre las razones 4,5:1 a 10:1.

Generalmente los tres criterios más importantes en la selección de las dimensiones de las vigas son: 
- Las tensiones en las alas.

- El costo de los materiales, y

- Una razonable estimación de la deformación.

Para aclarar el proceso de diseño, las fórmulas se presentan aplicadas al siguiente ejemplo de diseño:

Se desea dimensionar una viga doble-te para un galpón industrial de 4,8 $\mathrm{m}$ de luz. La viga presenta un sistema de carga conformado por el peso propio y dos carga de $300 \mathrm{Kg}$, cada una, ubicadas a los tercios de la luz.

La madera aserrada utilizada en la confección de las alas es de Pino radiata, Grado Estructural G1 y no presenta tratamiento químico alguno.

\section{Cuadro 1}

TENSIONES ADMISIBLES DEL PINO RADIATA, GRADO G1, SEGÚN NCH 1198.

\begin{tabular}{|l|c|c|}
\hline Madera aserrada & $\begin{array}{c}\text { Tensión admisible flexión } \\
\left(\mathrm{kg} / \mathrm{cm}^{2}\right)\end{array}$ & $\begin{array}{c}\text { Módulo de Elasticidad } \\
\left(\mathrm{kg} / \mathrm{cm}^{2}\right)\end{array}$ \\
\hline Pino radiata & 69 & 79.300 \\
\hline
\end{tabular}

Cuadro 2

PROPIEDADES MECÁNICAS DEL CONTRACHAPADO DE COIGÜE. ${ }^{(2)}$

\begin{tabular}{|l|c|c|c|}
\hline Contrachapado & $\begin{array}{c}\text { Tensión admisible flexión } \\
\left(\mathrm{kg} / \mathrm{cm}^{2}\right)\end{array}$ & $\begin{array}{c}\text { Módulo de } \\
\text { Elasticidad } \\
\left(\mathrm{kg} / \mathrm{cm}^{2}\right)\end{array}$ & $\begin{array}{c}\text { Tensión admisible de cizalle } \\
\left(\mathrm{kg} / \mathrm{cm}^{2}\right)\end{array}$ \\
\hline Coigüe & 140 & 105.500 & 13 \\
\hline
\end{tabular}




\section{DETERMINACIÓN DE LAS PROPIEDADES GEOMÉTRICAS DE LA SECCIÓN TRANSVERSAL}
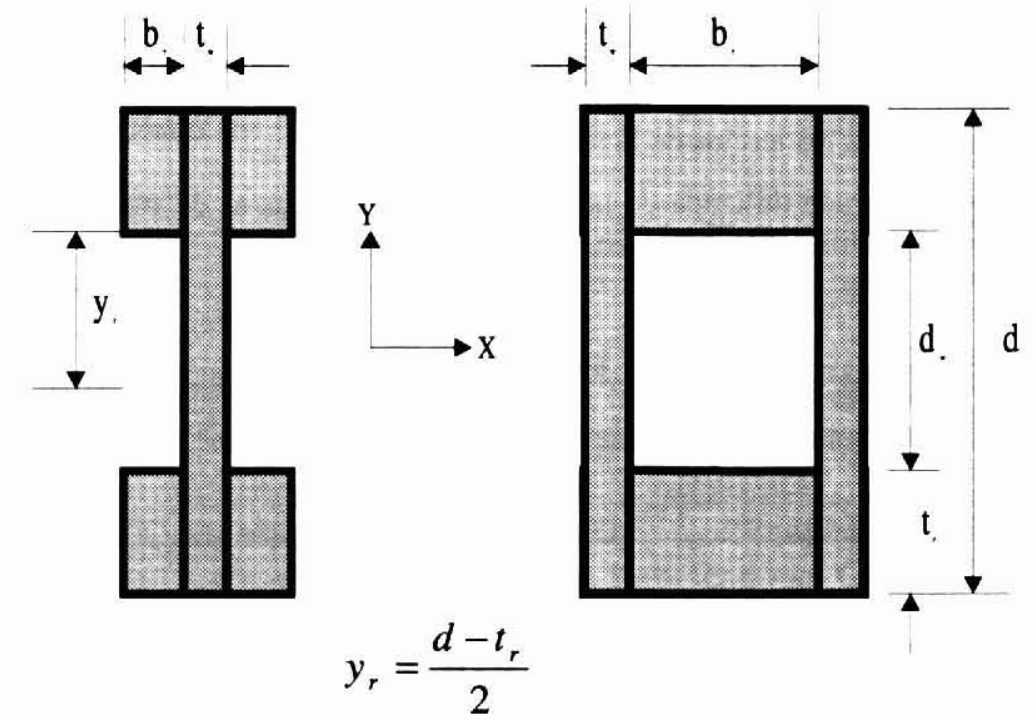

Figura 2. GEOMETRÍA DE LAS SECCIONES TRANSVERSALES.

Selección de una Sección Transversal Tentativa

Sea la altura de la viga $d=400 \mathrm{~mm}$, luego la razón $\mathrm{L} / \mathrm{d}=4800 / 40$ resulta ser igual a 12, encontrándose en el rango óptimo de 10 a 18.

Sea la sección de la viga de las siguientes características:

$$
\text { Alas : } t_{f}=9 \mathrm{~cm} \quad b_{f}=4,5 \mathrm{~cm} \quad \text { Ala: } t_{w}=9 \mathrm{~mm} \quad d_{w}=22 \mathrm{~cm}
$$

Contrachapado formado de 5 chapas, de las cuales 3 son paralelas a la luz de la viga $\left(k_{34}=3 / 5\right)$. 


\section{Momento de Inercia del Ala}

Se obtiene mediante el teorema de Steiner. Para una viga Cajón:

$$
\begin{gathered}
I_{x f}=\frac{b_{r}\left(d^{3}-d_{w}{ }^{3}\right)}{12} \\
I_{x f}=2 A_{r} y_{r}{ }^{2}
\end{gathered}
$$

o aceptando un porcentaje de error de $1 \%$ que se obtiene despreciando la inercia del ala respecto de su propio eje.

en que:

$$
A_{r}=b_{b} t_{r}
$$

Para vigas Doble Te, utilizando dos piezas de madera para cada ala, cada una de ellas de ancho $b_{f} y$ alto $t_{f}$, el valor de la inercia de las alas es:

$$
\begin{gathered}
I_{x f}=\frac{4}{12} b_{r} t_{r}^{3}+4 A_{r} y_{r}{ }^{2} \\
I_{x f}=\frac{4}{12} 4,5^{3} * 9^{3}+4 * 4,5 * 9 * 15,5^{2}=40.014 \mathrm{~cm}^{4}
\end{gathered}
$$

\section{Momento de Inercia del Alma}

El tablero de contrachapado presenta un comportamiento diferente al que presenta la madera compacta, en lo que a inercia se refiere, este comportamiento depende del número de chapas que constituye el tablero y de la dirección de las fibras de la cara exterior con respecto a la luz. Para la determinación de sus propiedades geométricas se considera el método de la sección efectiva que toma como colaborantes, en la determinación de las propiedades mecánicas, sólo a las chapas o tulipas del contrachapado que se disponen según la dirección de los esfuerzos principales. 


$$
I_{x w}=\frac{n k_{34} t_{w} d^{3}}{12}
$$

en que:

$$
\begin{aligned}
& n \text { = número de almas } \\
& n=1 \text { para vigas Doble Te } \\
& n=2 \text { para vigas Cajón } \\
& k_{34}=\text { Factor de chapas paralelas, que corresponde a la cantidad de } \\
& \text { chapas que se orientan paralela a la luz, respecto del total de } \\
& \text { chapas del tablero. } \\
& k_{34}=3 / 5 \text { para tablero de } 5 \text { chapas }(9 \mathrm{~mm} \text { ) } \\
& k_{34}=4 / 7 \text { para tablero de } 7 \text { chapas }(12 \mathrm{~mm}) \\
& k_{34}=5 / 9 \text { para tablero de } 9 \text { chapas }(15 \mathrm{~mm}) \\
& I_{x w}=\frac{1 *(3 / 5) * 0,9 * 40^{3}}{12}=2.880 \mathrm{~cm}^{4}
\end{aligned}
$$

\section{Rigidez en Flexión Respecto al Eje x-x}

La rigidez (EI) de una viga de sección transversal compuesta está constituida por el aporte que entregan las alas y el alma, en función de la geometría y su correspondiente Módulo de Elasticidad (MOE).

De esta manera, la EI respecto al eje $\mathrm{x}-\mathrm{x}$, es:

$$
(E I)_{x}=E_{f} I_{f x}+E_{w} I_{x w}
$$

donde:

$E_{f}=$ Módulo de elasticidad de la madera que conforma las alas.

$I_{x f}=$ Inercia de las alas respecto del eje $\mathrm{x}-\mathrm{x}$.

$\mathrm{E}_{\mathrm{w}}=$ Módulo de elasticidad del contrachapado.

$I_{x w}=$ Inercia del contrachapado del alma respecto al eje x-x.

Rigidez en Flexión Respecto al Eje y-y.

$$
(E I)_{x}=E_{x f}+E_{w} I_{y w}
$$


donde, para vigas Cajón se tiene:

$$
\begin{gathered}
I_{y f}=\frac{2 t_{f} b_{f}{ }^{3}}{12} \\
I_{y w}=\frac{k_{34} t_{w} d\left(b_{f}+t_{w}\right)^{3}}{2}
\end{gathered}
$$

Para Vigas Doble Te, despreciando conservadoramente la contribución del alma, lo cual lleva un error menor al $1 \%$, se tiene:

$$
I_{y f}=\frac{t_{r} b_{r}^{3}}{3}+t_{r} b_{r}\left(b_{r}+t_{w}\right)^{3}
$$

En este caso, la rigidez respecto al eje $\mathrm{X}-\mathrm{X}$ es:

$$
E I_{x}=E_{k} * I_{x}+E_{w} * I_{x w}=79.300 * 42.201+105.500 * 2,80=347,7 * 10^{7} \mathrm{~kg}^{*} \mathrm{~cm}^{2}
$$

\section{Momento del Área del Ala}

Usado para verificar la transferencia del cizalle en vigas clavadas.

Viga Cajón:

$$
Q_{x f}=\left(b_{r} t_{r}\right) \frac{\left(d-t_{r}\right)}{2}
$$

Viga Doble Te

$$
\begin{gathered}
Q_{x f}=2(4,5 * 9) \frac{(40-9)}{Q_{x f}}=2\left(b_{r} t_{r}\right) \frac{\left(d-255,5 \mathrm{~cm}^{3}\right.}{2} \\
Q_{t_{r}}
\end{gathered}
$$




$$
Q_{x f}=2(4,5 * 9) \frac{(40-9)}{2}=1.255,5 \mathrm{~cm}^{3}
$$

Momento del Área del Alma

$$
\begin{aligned}
& Q_{x w}=\frac{n k_{34} t_{w} d^{2}}{8} \\
& Q_{x w}=\frac{1 *(3 / 5) * 0,9 * 40^{2}}{8}=108 \mathrm{~cm}^{3}
\end{aligned}
$$

Momento del Área de Vigas

$$
\begin{aligned}
& (E Q)_{x}=E_{f} Q_{x f}+E_{w} Q_{x w} \quad E c .14 \\
& (E Q)_{x}=79.300 * 1255,5+105.500 * 108=1.110 .955 .150 \mathrm{~cm}^{3}
\end{aligned}
$$

\section{VERIFICACIÓN DE LA RESISTENCIA A LA FLEXIÓN}

\section{Momento Solicitante}

- Peso propio:

$$
\begin{array}{ll}
\text { Volumen de la viga } & =0,09504 \mathrm{~m}^{3} \\
\text { Densidad estimada de la viga } & =646 \mathrm{~kg} / \mathrm{m}^{3} \\
\text { Peso de la viga } & =61 \mathrm{~kg} \Rightarrow 13 \mathrm{~kg} / \mathrm{m}
\end{array}
$$

- Cargas puntuales:

$$
M=\frac{P}{2}
$$




\section{Tensión de Trabajo debida al Momento $M$}

Las tensiones inducidas en las alas, producto de la acción solicitante de un Momento de flexión, se traduce en esfuerzos de compresión y tracción, siendo éstas de mayor valor en las fibras más externas de la viga.

Para la determinación de estos esfuerzos existen dos metodologías: Una más analítica y otra aproximada. El método aproximado, usado para obtener un diseño estimado o para un primer cálculo antes de la verificación final, no toma la contribución del alma en la resistencia a la flexión. La carga actuante en el ala, sea ésta comprimida o traccionada corresponde a aquella que, con un brazo de palanca igual a la distancia entre centros de gravedad de las áreas consideradas, origina $\mathrm{el} \mathbf{M}_{\max }$.

$$
f_{b}=\frac{\text { Carga en el Ala }}{\text { Área del Ala }}=\frac{M_{\max } /\left(d-t_{r}\right)}{\left(b_{r}-t_{r}\right)}
$$

con: $f_{b}=$ Tensión de trabajo en las alas de tracción o compresión

Otra manera para determinar la tensión de trabajo, $\mathrm{f}_{\mathrm{b}}$, a que están sometidas las fibras más extremas de las alas y del contrachapado es con la conocida ecuación de Navier.

$$
f_{b}=\frac{M_{\text {máx }} y_{\text {máx }}}{I}
$$

con:

$$
y_{\max }=\frac{d}{2}
$$

Para vigas clavadas, es común usar como inercia el valor más conservador $I_{x f}$ aportado sólo por los elementos de las alas, despreciando el aporte del contrachapado del alma. Para vigas de contrachapado - madera, que han sido clavadas a lo largo de los ejes de las alas, las tensiones derivadas de la flexión (compresión y tracción), originan tensiones que son casi uniformes a través de la sección transversal del ala. Por lo tanto, para tales vigas clavadas el valor de $\mathbf{y}_{\max }$. puede sustituirse por el valor menos conservador $\mathrm{y}_{\mathrm{f}}$. El corrimiento de clavos puede producir una absorción del $10-15 \%$ del momento en el alma. Esto, normalmente, no produce problemas prácticos que considerar con vigas clavadas más livianas. 


\section{VERIFICACIÓN DEL CIZALLE DEL ALMA. DETERMINACIÓN DE LA TENSIÓN DE TRABAJO AL CIZALLE EN EL ALMA OCASIONADO POR LA CARGA DE CIZALLE}

Para la determinación del esfuerzo de corte V, la norma Chilena NCh 1198 establece que en las vigas soportadas por medio de un apoyo completo sobre un canto y con cargas aplicadas sobre el canto opuesto, se pueden despreciar todas las cargas ubicadas a una distancia no superior a la altura (h) de la pieza flexionada. Ver Figura 3.

Experiencias de otros países y el hecho que almas de contrachapado queden altamente solicitadas al cizalle, en los apoyos, sugieren que es prudente incluir todas las cargas ubicadas entre apoyos, cuando se verifique la capacidad de resistir cizalle.

En la práctica es normal tener rigidizadores de aplastamiento en los apoyos que transfieren parte de las cargas de cizalle directamente a éstos.

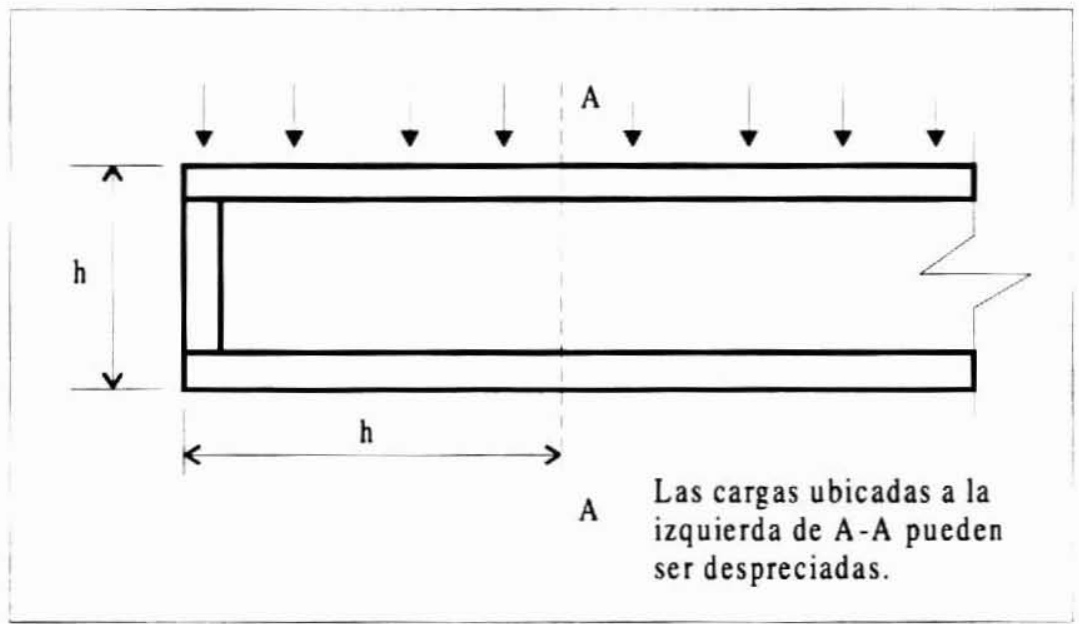

Figura 3. REPRESENTACIÓN DE LAS CARGAS DE CORTE A CONSIDERAR.

Si se requiere aumentar la capacidad de resistencia al cizalle del alma, es mejor incrementar el espesor del contrachapado o usar un contrachapado de 5 o más chapas. Una opción menos efectiva es usar, si está disponible, un contrachapado con mejor grado estructural. La única opción práctica es usar rigidizadores adicionales en el alma.

La tensión de trabajo al cizalle en el alma se puede obtener con la siguiente expresión: 


$$
f_{s}=\frac{V(E Q)_{x}}{(E I)_{x} n t_{w}}
$$

con:

$f_{s}=$ Tensión de trabajo al cizalle del alma.

$V \quad=$ Esfuerzo de corte.

$(E Q)_{x}=$ Momento del área de la viga.

$(E I)_{x}=$ Rigidez de la viga compuesta.

$n \quad=$ Número de almas de contrachapados que quedan resistiendo el cizalle.

$t_{w}=$ Espesor del contrachapado.

En el ejemplo:

$$
\begin{aligned}
& V=331 \mathrm{~kg} \\
& \qquad f_{s}=\frac{331 * 110.955 .15}{3.476 .950 .200 * 1 * 0,9}=11,7 \mathrm{~kg} / \mathrm{cm}^{2}
\end{aligned}
$$

En las almas del contrachapado, la distribución del cizalle es más lisa que la distribución parabólica de elementos sólidos. Una aproximación razonable se obtiene calculando la tensión de cizalle promedio, que induce a un error de aproximadamente un $10 \%$.

$$
f_{\text {S,prom. }}=\frac{V}{n t_{w} d}
$$

\section{DETERMINACIÓN DE LA TENSIÓN DE DISEÑo DE CIZALLE EN EL ALMA}

La tensión de diseño de cizalle del alma se puede obtener de la siguiente expresión:

$$
F^{\prime}{ }_{s}=K_{d} K_{H} F^{\prime}{ }_{s}
$$

donde:

$F_{S}^{\prime}=$ Tensión admisible de cizalle del contrachapado, dada por el cuadro 2. 
$K_{d}=$ Factor de modificación por duración de carga.

$K_{H}=$ Factor de modificación por humedad del contrachapado.

Para el ejemplo:

$$
F_{s}=1 * 1 * F^{\prime}{ }_{s}=13 \mathrm{~kg} / \mathrm{cm}^{2}>f_{s}
$$

\section{VERIFICACIÓN DE LAS DEFORMACIONES}

Dentro del rango de cargas de servicio, las vigas correctamente diseñadas y fabricadas con contrachapado se deflectan de acuerdo a la teoría de elasticidad pudiéndose emplear la fórmula estándar de deformación para calcular una parte de la deformación total, que es la causada por los esfuerzos de flexión.

El módulo de elasticidad en cizalle, llamado también módulo de rigidez, G, en la madera es relativamente bajo comparado con el módulo de elasticidad en flexión, con lo cual la deformación por corte puede ser una parte significativa de la deformación total.

El método para calcular la deformación de las vigas con madera-contrachapado queda expresado, en forma simple, con la ecuación:

$$
\Delta=\Delta b+\Delta s
$$

donde:

$\Delta b=$ Deformación por flexión de una viga.

$\Delta s=$ Deformación por cizalle.

Cuando existen varios tipos de carga actuando en una viga, la deformación máxima es la suma de las deformaciones calculadas para cada tipo de cargas actuando sola.

\section{DEFORMACIÓN POR FLEXIÓN}

Un método razonable exacto para calcular la deformación por flexión es la fórmula tradicional de deformación elástica de vigas sólidas para la carga aplicada, exceptuando que la rigidez seccional ( EI ) de la viga, corresponde a un valor compuesto por la suma de las rigideces de las alas y del contrachapado del alma.

Para el caso de viga simplemente apoyada, la deformación por flexión para el sistema de carga señalado en la figura siguiente, está dada por la siguiente ecuación: 


$$
\begin{aligned}
& \Delta b=\frac{(P / 2) a}{24 E Y}\left(3 I^{2}-4 a^{2}\right) \\
& \Delta b=\frac{3300 * 160}{24 * 3.476 .950 .200} *\left(3 * 480^{2}-4 * 160^{2}\right)=0,34 \mathrm{~cm}
\end{aligned}
$$

\section{DEFORMACIÓN POR CIZALLE}

Como el área del alma de contrachapado es baja respecto de la madera ocupada, entonces la deformación de cizalle puede resultar bastante alta. En madera, la deformación por cizalle es normalmente menor que el $5 \%$ de la deformación por flexión, sin embargo, en las vigas madera-contrachapado, la deformación por cizalle es alrededor de un $15 \%$ - $30 \%$ y puede resultar aún mayor.

Para cargas uniformemente distribuidas la Ec. 22 da una suficiente exactitud para estimar la deformación por cizalle, para luces simples, en la mitad de la luz y para una sección transversal con EI, constante.

$$
\Delta s=\frac{K_{\text {formaM }} M_{o}}{G A}
$$

A fin de evitar el cálculo del factor de forma $\left(K_{\text {forma }}\right)$, se propone por simplicidad una buena aproximación, consistente en tomar el valor de $K_{\text {forma }}=1$ y considerar como área, solamente el área del alma. Cualquier error que se manifieste es despreciable. Por lo tanto, la deformación por corte puede ser simplificada a:

$$
\Delta s=\frac{M_{o}}{G A_{s}}
$$

donde:

$\mathrm{M}_{\mathrm{O}}=$ Momento flector en la mitad de la luz de la viga.

$\mathrm{G}=$ Módulo de rigidez del contrachapado.

$\mathrm{A}_{\mathrm{S}}=$ Area cizallada . 
$A_{S}=2 t_{w} d_{N} \quad$ para vigas cajón.

$A_{S}=t_{w} d_{N} \quad$ para vigas doble te.

$\mathrm{d}_{\mathrm{N}}=$ altura entre el clavado superior e inferior en la unión ala-alma

$$
\Delta_{s}=\frac{51.744}{5.275 * 31,5}=0,31 \mathrm{~cm}
$$

Por lo tanto la deformación total es $0,65<\mathrm{L} / 360=480 / 360=1,33 \mathrm{~cm}$

\section{DISEÑO DE LA UNION ALA - ALMA}

Cálculo del Flujo de Cizalle.

Según NCh 1198:

$$
q=\frac{V *(E Q)_{x f}}{(E I)_{x}} * \frac{1}{n}=\frac{330 * 99.561 .150}{3.476 .950 .200} * \frac{1}{2}=4,72 \mathrm{~kg} / \mathrm{cm}
$$

\section{Cálculo de la Carga Admisible de Extracción Lateral del Clavo, $\mathbf{P}_{\text {adm }}$}

Dimensiones del clavo a usar: $\mathrm{dc}=3,5 \mathrm{~mm}$ (diámetro del clavo)

$$
\mathrm{lc}=75 \mathrm{~mm} \text { (largo del clavo). }
$$

Densidad anhidra de la madera (Pino radiata) $\rho_{\mathrm{o}, \mathrm{k}}=369 \mathrm{~kg} / \mathrm{m}^{3}$

$$
P_{e l, a d m}=3,5 * d c^{1.5} * \rho^{0.5}=3,5 * 3,5^{1.5} * 369^{0.5}=440 \mathrm{~N}
$$

La carga admisible de extracción lateral para el clavo en cizalle doble es:

$$
P_{a d m}^{(\bullet)}=\left((m-1)+0,75 * K_{p p} * P_{e l, a d m}=((2-1)+0,75 * 0,75 * 440=687, N / \text { clavo }\right.
$$

Por lo tanto el máximo espaciamiento entre clavos es:

$$
P_{\text {adm }}^{(*)}=68,75 \mathrm{~kg} / \text { clavo }
$$




$$
s=\frac{P_{a d m}^{(*)}}{q}=14 \mathrm{~cm} / \text { clavo }
$$

(*) Se adoptará un espaciamiento real de $10 \mathrm{~cm}$ en doble fila.

(*) Se colocarán rigidizadores en los extremos de la viga e intermedios, separados en 120 $\mathrm{cm}$, uno del otro, siendo éste de la misma escuadría de los elementos que forman las alas.

FABRICACIÓN Y ENSAYO DE VIGAS DOBLE TE Y VIGAS CAJÓN. RELACIÓN Y DETERMINACIÓN DE PARÁMETROS A INCLUIR EN EL ESTUDIO.

La finalidad de este estudio es la verificación experimental de la resistencia de vigas de madera de sección transversal compuesta obtenida mediante el método teórico propuesto.

En la tabla siguiente se incluyen los elementos y parámetros que se usaron en la confección de las distintas vigas.

Cuadro 3

ELEMENTOS USADOS EN LA CONFECCIÓN DE LAS VIGAS.

\begin{tabular}{|c|c|c|c|c|}
\hline $\begin{array}{l}\text { PERFIL } \\
\text { VIGA } \\
\end{array}$ & UNIÓN & ELEMENTO & $\begin{array}{c}\text { ESPECIE } \\
\text { MADERERA }\end{array}$ & $\begin{array}{l}\text { DIMENSIONES } \\
(\mathrm{mm})\end{array}$ \\
\hline \multirow{2}{*}{$\begin{array}{l}\text { DOBLE } \\
\text { TE }\end{array}$} & \multirow{2}{*}{$\begin{array}{l}\text { Clavada } \\
\text { y } \\
\text { Clavada - } \\
\text { Encolada }\end{array}$} & ALA & Pino radiata & $\begin{array}{c}* 4 \text { Listones de } \\
45 * 90 * 3200 \\
* 4 \text { Listones de } \\
45 * 90 * 1600\end{array}$ \\
\hline & & ALMA & Coigüe & $\begin{array}{c}* 2 \text { Tableros de } \\
\text { e-*400*2400 } \\
(\mathrm{e}=9 ; 12 \text { y } 15 \mathrm{~mm})\end{array}$ \\
\hline \multirow[t]{2}{*}{ CAJÓN } & \multirow{2}{*}{$\begin{array}{l}\text { Clavada } \\
\text { y } \\
\text { Clavada - } \\
\text { Encolada }\end{array}$} & ALA & Pino radiata & $\begin{array}{c}* 2 \text { Listones de } \\
45 * 90 * 3200 \\
* 2 \text { Listones de } \\
45 * 90 * 1600\end{array}$ \\
\hline & & ALMA & Coigüe & 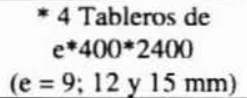 \\
\hline
\end{tabular}

NOTA: El total de vigas ensayadas fue:

Tipos de vigas 2

Tipo de unión 2

Espesor alma. $\quad 3$

Repeticiones 9

TOTAL: $2 * 2 * 3 * 9=108$ 


\section{NOMENCLATURA USADA}

Para facilitar la identificación individual de cada viga, se empleó la nomenclatura que a continuación se detalla.

donde:

$$
\begin{aligned}
\text { DT }-\mathrm{i}-\mathrm{c}-\mathrm{k} & \text { para vigas DOBLE TE } \\
\mathrm{C}-\mathrm{i}-\mathrm{ce}-\mathrm{k} & \text { para vigas CAJON }
\end{aligned}
$$

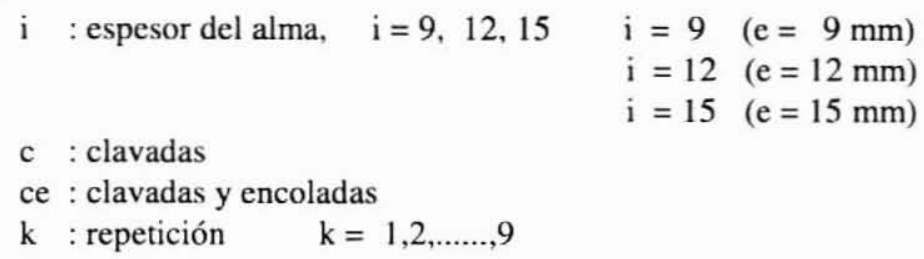

EJEMPLO: $\quad$ DT - 12 - ce - 8, significa:

$$
\begin{aligned}
& \text { DT: Sección DOBLE TE } \\
& 12: \text { espesor del alma } \mathrm{e}=12 \mathrm{~mm} \\
& \text { ce : clavada y encolada } \\
& 8 \text { : octava repetición. }
\end{aligned}
$$

La verificación del número de ensayos necesarios, se realizó considerando un nivel de confiabilidad de $95 \%$.

Se comprobó que la cantidad de ensayos realizados ( 9 por cada tipo de sección) fue suficiente, siendo esta cantidad superior al mínimo necesario para el nivel de confianza establecido.

\section{DESCRIPCIÓN DEL ENSAYO DE FLEXIÓN}

El ensayo de flexión consiste básicamente en medir la deformación que se produce en el centro de la luz de una viga simplemente apoyada en sus extremos, al aplicar carga sobre ella. Esta carga aumenta paulatinamente en el tiempo hasta llegar a la ruptura de la viga.

Una vez instalada la viga se le adosan todos los instrumentos necesarios para el ensayo, se calibra el programa computacional a través de una serie de parámetros que tienen que ver con la carga y deformación esperada, además del intervalo de tiempo deseado en que el programa irá registrando todos los datos que se desean obtener del ensayo. El computador, simultáneamente, muestra el gráfico Carga-Deformación, además de capturar aquellos valores que forman dicho gráfico. 


\section{ANALISIS Y COMPARACIÓN DE RESULTADOS TEÓRICOS VERSUS EXPERIMENTALES}

Como se dijo anteriormente, el objetivo de este estudio es determinar experimentalmente, a través de un ensayo de flexión, las propiedades mecánicas de las vigas Doble $\mathrm{Te}$ y Cajón, construidas con madera aserrada de Pino radiata y contrachapado de Coigüe, de tal manera de poder comparar dichos resultados con aquellos que señala el procedimiento teórico propuesto.

En el análisis teórico se considera una viga conformada por alas de calidad estructural G1 ( NCh 1207 ). En la realidad las vigas fueron fabricadas con grados de esta calidad o mejor, calidades que dada la forma visual con que fueron clasificadas, están sujetas a un error de apreciación y cierta subjetividad.

En la determinación teórica de las propiedades resistentes, se considera un diseño para cargas de 10 años de duración, para lo cual se aplican los factores de modificación correspondientes y que se encuentran detallados en la norma NCh 1198

\section{DETERMINACIÓN TEÓRICA DE LA TENSIÓN DE DISEÑO DE FLEXIÓN}

La tensión de diseño en flexión para el Pino radiata se determina de acuerdo a la metodología especificada en la norma chilena NCh 1198.

De acuerdo a lo anterior y considerando una viga con alas de Pino radiata en estado seco y grado estructural $\mathrm{G} 1$, la tensión admisible en flexión $\left(\mathrm{F}_{\mathrm{f}}\right)$, tiene un valor de 69 $\mathrm{kg} / \mathrm{cm}^{2}$. Ver cuadro 1 .

Cabe señalar que debido al arriostramiento lateral dispuesto, durante el ensayo, no existe reducción de la tensión admisible en el ala flexo-comprimida, como tampoco existe reducción en el ala flexo-traccionada.

Por lo tanto, la tensión de diseño en flexión del Pino radiata, tanto para la viga DOBLE TE como para la viga CAJÓN toma el valor de $69 \mathrm{~kg} / \mathrm{cm}^{2}$.

\section{DETERMINACIÓN TEÓRICA DE LA CARGA DE DISEÑO}

La carga teórica de diseño, corresponde a la carga máxima que puede soportar la viga bajo un sistema de solicitación determinado, sin sobrepasar la tensión de diseño que la metodología establece.

Para determinar esta carga máxima, es necesario conocer: el sistema de combinación de cargas, forma en que se dispone la viga, condiciones de apoyo y disposición de las cargas, en el momento del ensayo.

El sistema utilizado, en este estudio, se incluye en la figura siguiente. 


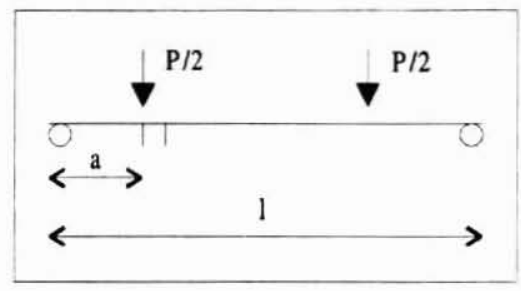

FIGURA 4. DISPOSICIÓN Y SISTEMA DE CARGA DE LA VIGA ESTUDIADA.

Para conocer cual será la carga teórica de flexión, previamente es necesario determinar el momento solicitante y la inercia correspondiente a cada una de las secciones transversales en estudio, valores que a continuación se incluyen.

\section{DETERMINACIÓN TEÓRICA DE LA CARGA MÁXIMA POR FLEXIÓN}

\section{Cálculo del Momento Solicitante}

En la determinación del Momento flector, se considera, además del efecto de las cargas puntuales, el efecto que produce el peso propio de la viga.

$$
\begin{aligned}
& M=177\left(\frac{P}{2}\right)+\frac{q 464^{2}}{8}(\mathrm{kgcm}) \\
& M=177 *(300)+\frac{* 464^{2}}{8}(\mathrm{~kg} * \mathrm{~cm})
\end{aligned}
$$

\section{Cálculo de las Inercias}

Según la metodología propuesta y obedeciendo a un diseño más conservador, se considera que son las alas los elementos capaces de soportar los esfuerzos debidos a la flexión, de ahí que la inercia que interesa en la determinación de la tensión debida al momento, es la inercia $\mathrm{I}_{\mathrm{xf}}$ de las alas, calculada según Ec.1 a Ec. 4.

Conocida la tensión de diseño en flexión para el Pino radiata, es posible, por medio la ecuación de Navier, determinar la carga máxima que resisten las vigas, en flexión. 


$$
\frac{M_{\text {max }} * y_{\text {max }}}{I_{x f}}=F_{f, \text { dis }}
$$

con:

$$
y_{\text {mix }}=20 \mathrm{~cm} \quad F_{\text {f.dis }}=69,0 \mathrm{~kg} / \mathrm{cm}^{2}
$$

De esta manera se tiene que para cada una de las secciones transversales de las vigas estudiadas, las cargas máximas admisibles para flexión, son las incluidas en el cuadro 4.

Cuadro 4

DETERMINACIÓN TEÓRICA DE LA CARGA MÁXIMA ADMISIBLE PARA FLEXIÓN, EN LAS VIGAS ESTUDIADAS.

\begin{tabular}{|c|c|c|c|c|}
\hline VIGA & $\begin{array}{c}\text { Peso viga } \\
(\mathrm{kg})\end{array}$ & $\begin{array}{c}\text { Largo efectivo } \\
(\mathrm{cm})\end{array}$ & $\begin{array}{c}\text { Peso propio } \\
\mathbf{q} \\
(\mathrm{kg} / \mathrm{m})\end{array}$ & $\begin{array}{c}\text { Plexion } \\
(\mathrm{kg})\end{array}$ \\
\hline DT $9 \mathrm{~mm}$ & 55,9 & 464 & 12,0 & 1.523 \\
\hline DT $12 \mathrm{~mm}$ & 61,0 & 464 & 13,1 & 1.520 \\
\hline DT $15 \mathrm{~mm}$ & 73,8 & 464 & 15,9 & 1.511 \\
\hline C $9 \mathrm{~mm}$ & 49,7 & 464 & 10,7 & 967 \\
\hline C $12 \mathrm{~mm}$ & 56,0 & 464 & 12,1 & 963 \\
\hline C $15 \mathrm{~mm}$ & 65,6 & 464 & 14,1 & 957 \\
\hline
\end{tabular}

\section{DETERMINACIÓN TEÓRICA DE LA CARGa MÁXIMA ADMISIBLE POR CIZALLE DEL ALMA}

Con el valor de la tensión de diseño de cizalle del contrachapado del alma, la que tiene un valor de $13 \mathrm{~kg} / \mathrm{cm}^{2}$ ( Ver Cuadro 2), es posible conocer la carga máxima que la viga, sin sobrepasar la tensión anterior, puede soportar trabajando al corte.

Haciendo uso de la Ec. 6 y de la Ec 14 es posible determinar dicha carga $\left(P_{\text {cizalle }}\right.$, la que a continuación se calcula para cada tipo de viga. 
Cuadro 5

DETERMINACIÓN DE LA CARGA MÁXIMA ADMISIBLE POR CIZALLE DEL CONTRACHAPADO DEL ALMA.

\begin{tabular}{|c|c|c|c|}
\hline VIGA & $\begin{array}{c}(\mathrm{EQ}) \mathrm{x} \\
\left(\mathrm{kg}^{*} \mathrm{~cm}\right)\end{array}$ & $\begin{array}{c}(\mathrm{EI})_{\mathrm{X}} \\
\left(\mathrm{kg}^{*} \mathrm{~cm}^{2}\right) \\
\end{array}$ & $\begin{array}{l}P_{\text {cizalle }} \\
(\mathrm{kg})\end{array}$ \\
\hline DT $9 \mathrm{~mm}$ & 110.955 .150 & 3.476 .950 .200 & 733 \\
\hline DT $12 \mathrm{~mm}$ & 114.025 .200 & 3.558 .923 .700 & 974 \\
\hline DT $15 \mathrm{~mm}$ & 117.148 .000 & 3.641 .952 .200 & 1.212 \\
\hline C $9 \mathrm{~mm}$ & 79.796 .770 & 2.642 .256 .310 & 1.549 \\
\hline C $12 \mathrm{~mm}$ & 85.947 .420 & 2.806 .203 .310 & 2.037 \\
\hline C $15 \mathrm{~mm}$ & 92.171 .920 & 2.972 .260 .310 & 2.515 \\
\hline
\end{tabular}

\section{DETERMINACIÓN TEÓRICA DE LA CARGA MÁXIMA ADMISIBLE POR CONDICIÓN DE DEFORMACIÓN MAXIMA ADMISIBLE}

La deformación máxima admisible (condición de estética) para una viga de madera, está dada por $\delta=\mathrm{L} / 300$. Para la viga en diseño, esta deformación admisible tiene un valor de $1,6 \mathrm{~cm}$.

De las Ec. 24 , Ec. 25 y Ec. 26, se deduce que la carga máxima admisible por condición de deformación $\left(\mathrm{P}_{\text {def }}\right)$ está dada por la ecuación:

$$
1,6=\frac{P_{\text {def }} * 177 *\left(3 * 480^{2}-4 * 177^{2}\right)}{48 * E I_{x}}+\frac{\left(P_{\text {def }} / 2\right) * 177+q *\left(480^{2} / 8\right)}{5257 * A_{s}} E c .26
$$

Para cada una de las secciones estudiadas, se obtienen las siguientes cargas máximas admisibles por deformación.

\section{Cuadro 6}

DETERMINACIÓN DE LAS CARGAS MÁXIMAS ADMISIBLES POR CONDICIÓN DE DEFORMACIÓN.

\begin{tabular}{|c|c|c|c|c|}
\hline VIGA & $\begin{array}{c}\text { Elx } \\
\left(\mathrm{kg}^{*} \mathrm{~cm}^{2}\right)\end{array}$ & $\begin{array}{c}\mathrm{q} \\
(\mathrm{kg} / \mathrm{m})\end{array}$ & $\begin{array}{c}\text { As } \\
\left(\mathrm{cm}^{2}\right)\end{array}$ & $\begin{array}{c}P_{\text {def }} \\
(\mathrm{kg})\end{array}$ \\
\hline DT 9 mm & 3.476 .950 .200 & 12,0 & 31,5 & 1.394 \\
\hline DT $12 \mathrm{~mm}$ & 3.558 .923 .700 & 13,1 & 42,0 & 1.606 \\
\hline DT $15 \mathrm{~mm}$ & 3.641 .952 .200 & 15,9 & 52,5 & 1.774 \\
\hline C $9 \mathrm{~mm}$ & 2.642 .256 .310 & 10,7 & 63,0 & 1.506 \\
\hline C $12 \mathrm{~mm}$ & 2.806 .203 .310 & 12,1 & 84,0 & 1.688 \\
\hline C $15 \mathrm{~mm}$ & 2.972 .260 .310 & 14,1 & 105,0 & 1.847 \\
\hline
\end{tabular}




\section{DETERMINACIÓN TEÓRICA DE LA CARGA DE DISEÑO PARA LA VIGA}

La carga de diseño de la viga corresponde a la mayor carga que ésta es capaz de soportar, sin fallar ninguno de sus elementos constituyentes.

Corresponde a la menor de las cargas máximas de flexión, cizalle y deformación obtenidas e incluidas en los cuadros 4,5 y 6 .

A continuación se presentan las cargas máximas que soportan los tipos de vigas a las solicitaciones de flexión, cizalle y por condición de deformación, destacándose la carga que limita el diseño.

\section{Cuadro 7}

\section{COMPARACIÓN DE LAS CARGAS MÁXIMAS OBTENIDAS PARA SOLICITACIONES DE FLEXIÓN, CIZALLE Y DEFORMACIÓN.}

\begin{tabular}{|c|c|c|c|c|}
\hline $\begin{array}{c}\text { VIGA } \\
\text { TIPO }\end{array}$ & $\begin{array}{c}P_{\text {flex }} \\
(\mathrm{kg})\end{array}$ & $\begin{array}{c}\mathrm{P}_{\mathrm{ciz}} \\
(\mathrm{kg})\end{array}$ & $\begin{array}{c}\mathrm{P}_{\text {deform }} \\
(\mathrm{kg})\end{array}$ & $\begin{array}{c}\text { Diseño limitado } \\
\text { por }\end{array}$ \\
\hline DT $9 \mathrm{~mm}$ & 1.523 & $\mathbf{7 3 3}$ & 1.394 & cizalle contrachapado \\
\hline DT $12 \mathrm{~mm}$ & 1.520 & $\mathbf{9 7 4}$ & 1.688 & cizalle contrachapado \\
\hline DT $15 \mathrm{~mm}$ & 1.511 & $\mathbf{1 . 2 1 2}$ & 1.774 & cizalle contrachapado \\
\hline C $9 \mathrm{~mm}$ & $\mathbf{9 6 7}$ & 1.549 & 1.506 & flexión alas \\
\hline C $12 \mathrm{~mm}$ & $\mathbf{9 6 3}$ & 2.037 & 1.688 & flexión alas \\
\hline C $15 \mathrm{~mm}$ & $\mathbf{9 5 7}$ & 2.515 & 1.847 & flexión alas \\
\hline
\end{tabular}

Por lo tanto, de acuerdo a lo anterior, las cargas de diseño teóricas para cada una de las secciones transversales en estudio son las incluidas en el cuadro 8.

Cuadro 8

CARGA DE DISEÑO TEORICO PARA LAS SECCIONES TRANSVERSALES DE LAS VIGAS EN ESTUDIO.

\begin{tabular}{|c|c|}
\hline \begin{tabular}{|c|} 
VIGA \\
TIPO
\end{tabular} & $\begin{array}{c}\text { Pdis, tornica } \\
(\mathrm{kg})\end{array}$ \\
\hline DT $9 \mathrm{~mm}$ & 733 \\
\hline DT $12 \mathrm{~mm}$ & 974 \\
\hline DT $15 \mathrm{~mm}$ & 1.212 \\
\hline C $9 \mathrm{~mm}$ & 967 \\
\hline C $12 \mathrm{~mm}$ & 963 \\
\hline C $15 \mathrm{~mm}$ & 957 \\
\hline
\end{tabular}




\section{TENSIÓN ADMISIBLE DE FLEXIÓN, DETERMINADA EXPERIMENTALMENTE}

La tensión admisible en flexión, determinada experimentalmente, se obtiene de los ensayos, de los diferentes tipos de vigas a escala real, en los cuales es posible determinar las cargas y deformaciones máximas alcanzadas por cada viga. La obtención de estos valores se realiza en aquella sección transversal de la viga donde se presenta la menor capacidad resistente de ella. Ver Figura 5. Cabe hacer notar que esta sección crítica de la viga coincide con la sección en la cual ocurrió la falla durante el ensayo.

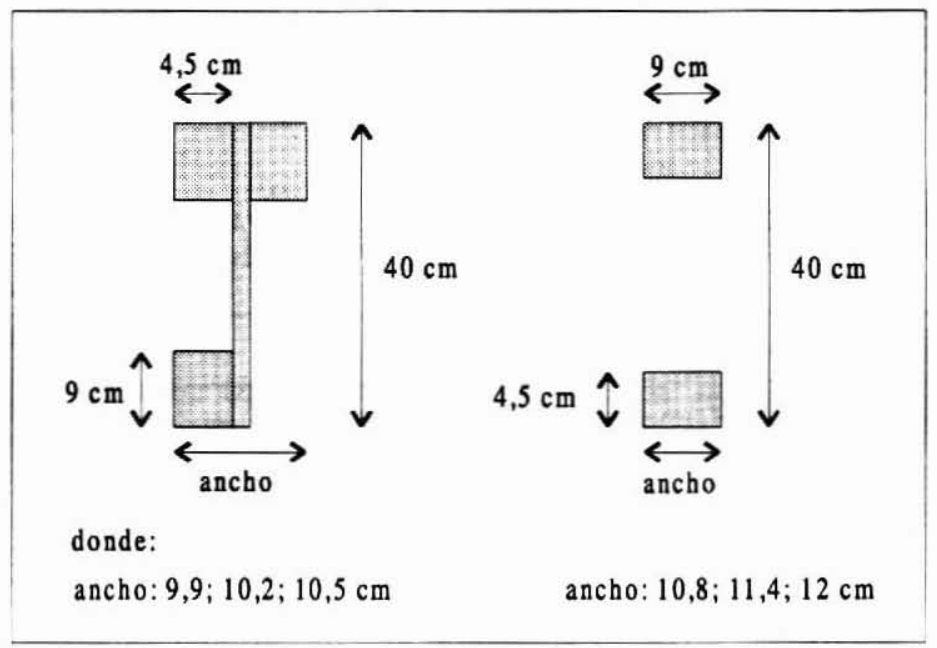

Figura 5. SECCIÓN CRÍTICA, QUE CORRESPONDE A LA DISCONTINUIDAD TANTO EN LAS ALAS DE LA VIGA dOBLE TE COMO EN EL CENTRO dE LA VIGA CAJÓN.

Conocida la carga máxima capaz de soportar cada viga, es posible encontrar el promedio de estas cargas obtenido para un determinado tipo de viga, su desviación estándar, el coeficiente de variación y el valor mínimo que se obtiene con una confiabilidad del $95 \%$.

Este valor límite de resistencia, $\mathbf{P}_{\mathrm{l} \text { lim }}, \mathbf{5} \%$, se denomina "límite inferior con exclusión de $5 \%$ “, y él asegura, con una certeza de $95 \%$, que la resistencia de cualquier viga similar a las ensayadas, será superior al límite de resistencia elegido como base. La carga admisible ( $\mathbf{P}_{\text {adm }}$ ) se obtiene de este valor de resistencia límite, el cual es dividido por un factor de ajuste "n" que incluye una corrección para considerar la aplicación de una carga de duración prolongada (10 años), un trabajo elástico y un factor de seguridad. 
En flexión, este factor de ajuste toma el valor: $\mathbf{n}=\mathbf{2 , 1}$. Por medio de la siguiente expresión es posible encontrar la carga admisible para cada tipo de sección transversal estudiada:

$$
P_{\text {adm }}=\frac{P_{\mathrm{lim}, 5 \%}}{n}
$$

Con:

$$
P_{\text {lim, } 5 \%}=P-1,645 * S_{p}
$$

en donde:

$\mathrm{P} \quad$ = Carga de ruptura promedio del tipo de viga analizado.

$\mathrm{S}_{\mathrm{P}} \quad=$ Desviación estándar de las cargas obtenidas para el tipo de viga en estudio.

\section{DATOS OBTENIDOS EN LOS ENSAYOS DE LOS TIPOS DE VIGAS ENSAYADAS}

El cuadro siguiente entrega las tensiones admisibles calculadas de los datos experimentales para cada uno de los tipos de secciones estudiados.

\section{Cuadro 9}

\begin{tabular}{|c|c|c|c|c|c|}
\hline \multirow[t]{2}{*}{ VIGA } & \multirow[t]{2}{*}{ UNIÓN } & \multicolumn{3}{|c|}{$\begin{array}{c}\text { MODULO DE RUPTURA } \\
\left(\mathrm{kg} / \mathrm{cm}^{2}\right)\end{array}$} & \multirow{2}{*}{$\begin{array}{c}\text { TENSION } \\
\text { ADM. } \\
\text { EXPERIM. } \\
\left(\mathrm{kg} / \mathrm{cm}^{2}\right)\end{array}$} \\
\hline & & PROMEDIO & $\begin{array}{c}\text { DESV. } \\
\text { ESTANDAR }\end{array}$ & $\mathrm{R}_{\mathrm{MIN}, 5 \%}$ & \\
\hline \multirow{3}{*}{$\begin{array}{l}\text { DT-09-C } \\
\text { DT-12-C } \\
\text { DT-15-C }\end{array}$} & \multirow{3}{*}{ Clavada } & 174,2 & 14,3 & 150,6 & 71,7 \\
\hline & & 176,0 & 14,4 & 152,3 & 72,5 \\
\hline & & 181,0 & 23,9 & 142,3 & 67,8 \\
\hline \multirow{3}{*}{$\begin{array}{l}\text { DT-09-CE } \\
\text { DT-12-CE } \\
\text { DT-15-CE }\end{array}$} & \multirow{3}{*}{$\begin{array}{c}\text { Clavada } \\
y \\
\text { Encolada } \\
\end{array}$} & 159,3 & 29,5 & 110,8 & 52,8 \\
\hline & & 176,4 & 30,1 & 126.9 & 60,4 \\
\hline & & 163,7 & 31,9 & 111,2 & 53,0 \\
\hline \multirow{3}{*}{$\begin{array}{l}\text { C-09-C } \\
\text { C-12-C } \\
\text { C-15-C }\end{array}$} & \multirow{3}{*}{ Clavada } & 134,9 & 13,2 & 113,2 & 53,9 \\
\hline & & 146,3 & 27,3 & 101,4 & 48,3 \\
\hline & & 184,6 & 29,2 & 136,6 & 65,0 \\
\hline C-09-CE & \multirow{3}{*}{$\begin{array}{l}\text { Clavada } \\
\text { y } \\
\text { Encolada }\end{array}$} & 161,1 & 30,4 & 111,1 & 52,9 \\
\hline C-12-CE & & 158,2 & 6,8 & 147,0 & 70,0 \\
\hline C-15-CE & & 254,1 & 9,0 & 239,3 & 114,0 \\
\hline
\end{tabular}

DETERMINACIÓN DE LA TENSIÓN ADMISIBLE DE FLEXIÓN, EXPERIMENTAL. 


\section{CONCLUSIONES}

Del manejo, elaboración y estudio estadístico de los datos experimentales, se obtienen las siguientes conclusiones:

\section{Características Físicas}

1.1. Las vigas con sección transversal Doble-te (DT), tanto clavadas como clavadasencoladas, tenían un peso promedio de 12,$0 ; 13,1$ y $15,9 \mathrm{~kg} / \mathrm{ml}$, usando en su fabricación espesor de contrachapado de $9 ; 12$ y $15 \mathrm{~mm}$, respectivamente.

1.2. Las vigas con sección transversal Cajón (C), tanto clavadas como clavadasencoladas, en promedio, pesaban 10,$7 ; 12,1$ y $14,1 \mathrm{~kg} / \mathrm{ml}$, al ser fabricadas con espesores de contrachapado de 9,12 y15 mm, respectivamente.

1.3. Las vigas DT, en promedio, presentan un mayor peso en cada uno de los espesores de contrachapado, respecto a las vigas C. Porcentualmente, este mayor peso es de: $12,1 \% ; 8,3 \%$ y $12,8 \%$, respectivamente, para los espesores de $9 ; 12$ y $15 \mathrm{~mm}$.

1.4. La madera de Pino radiata, que conformaba las alas de las vigas, tenía un contenido de humedad promedio de $12 \%$.

1.5. El contrachapado de Coigüe, que conformaba el alma de las vigas, presentó un contenido de humedad de $10 \%$, para todos los espesores.

1.6. El promedio de la razón entre los dos Momentos de Inercia obtenidos en los Ejes Principales de Inercia (ix/iy) para la viga Doble-te, resultó ser 22,5; 21,5 y 20,5 para los espesores de contrachapado de $9 ; 12$ y $15 \mathrm{~mm}$, respectivamente.

1.7. El promedio de la razón entre los dos Momentos de Inercia obtenidos en los Ejes Principales de Inercia (ix/iy) para la viga Cajón, resultó ser 10,2; 8,1 y 6,5 para los espesores de contrachapado de 9,12 y $15 \mathrm{~mm}$, respectivamente.

\section{Propiedades Mecánicas de las Vigas Ensayadas}

2.1 Al comparar la Tensión Admisible Teórica (TAT) con la Tensión Admisible Experimental (TAE) resultante para cada sección transversal, en cada tipo de unión, se concluye que los $2 / 3$ de las TAE resultaron menores que la TAT resultante del proceso de cálculo teórico.

Para ajustar ambos procedimientos se recomienda el uso del siguiente Factor de Modificación por forma y tipo de unión $\left(\mathrm{K}_{\mathrm{F}, \mathrm{U}}\right)$, aplicable a la TAT obtenida según las especificaciones de la NCh 1198. 
Cuadro 10

\section{FACTOR DE MODIFICACIÓN POR FORMA Y UNIÓN.APLICABLE A LA TENSIÓN ADMISIBLE TEÓRICA.}

\begin{tabular}{|l|c|c|}
\hline \multirow{2}{*}{ TIPO DE UNIÓN } & $\begin{array}{c}\text { SECCIÓN } \\
\text { TRANSVERSAL }\end{array}$ & K$_{\mathrm{UF}}$ \\
\hline CLAVADA & DT & 0,98 \\
\hline CLAVADA Y & $\mathrm{C}$ & 0,70 \\
\cline { 2 - 3 } ENCOLADA & DT & 0,76 \\
\cline { 2 - 3 } & $\mathrm{C}$ & 0,76 \\
\hline
\end{tabular}

NOTA: Este Factor de Modificación se debe aplicar a vigas compuesta con secciones transversales Doble-te y Cajón, fabricadas en forma similar a las contempladas en este estudio. Estas tenían alas con uniones de tope debido a que la intención era probar, en esta etapa, las vigas confeccionadas utilizando herramientas simples tales como martillo, serrucho y brocha. En una etapa posterior se contemplarán uniones más firmes, para las piezas que forman las alas, como son las uniones endentadas, encoladas y placas metálicas endentadas.

2.2. En general, el diseño de las vigas incluidas en este trabajo queda controlado por la deformación máxima admisible $\left(\delta_{\text {máx, adm }}\right)$

2.3. Todas las Tensiones Admisibles Experimentales obtenidas en la totalidad de las vigas estudiadas se ubican en la zona de comportamiento elástico del elemento estructural.

2.4. El valor de la Tensión Admisible calculado teóricamente se ubica en la zona de comportamiento elástico, obtenida experimentalmente, en todas las vigas ensayadas, alcanzando los siguientes porcentajes de la Tensión en el Límite de Proporcionalidad, para los distintos tipos de vigas:

i ) En vigas Doble-te clavadas: $46 \% ; 45 \%$ y $49 \%$ para espesores de contrachapados de $9 ; 12$ y $15 \mathrm{~mm}$, respectivamente.

ii ) En vigas Cajón clavadas: $61 \%$; $68 \%$ y $51 \%$ para espesores de contrachapado de $9 ; 12$ y $15 \mathrm{~mm}$, respectivamente.

iii) En vigas Doble-te clavadas y encoladas: $62 \%$; $54 \%$ y $51 \%$ para espesores de contrachapados de 9: 12 y $15 \mathrm{~mm}$, respectivamente.

iv) En vigas Cajón clavadas y encoladas: $62 \% ; 47 \%$ y $29 \%$ para espesores de contrachapados de $9 ; 12$ y $15 \mathrm{~mm}$, respectivamente.

2.5. Para todas las vigas la Rigidez, $(\mathrm{EI})_{\text {teo. }}$ obtenida teóricamente, resultó mayor que aquella obtenida experimentalmente. Luego, las deformaciones que entrega el proceso teórico resultan menores que las reales, determinadas experimentalmente. 
2.6. Entre las secciones transversales estudiadas, la más conveniente resulta ser la viga Doble-te Clavada pues tiene menor peso propio, menor costo, da plena confianza de trabajo elástico, puede arriostrarse con comodidad, etc.

Respecto a la mayor economía que se logra con la viga Doble-te, basta revisar la participación de los distintos materiales constituyentes, en el peso de ambos tipos de vigas.

MATERIAL

Madera aserrada

Contrachapado

Clavos
VIGA DOBLE-TE

$75 \%$

$21 \%$

$4 \%$
VIGA CAJÓN

$50 \%$

$47 \%$

$3 \%$

La incidencia del contrachapado es preponderante en el mayor costo que se obtiene en las vigas Cajón, para una misma luz y altura.

2.7. El método de cálculo presentado permite, con la introducción del Factor de Modificación $\left(\mathrm{K}_{\mathrm{UF}}\right)$, un diseño que establece un buen acercamiento entre lo teórico y lo experimental

\section{REFERENCIAS BIBLIOGRÁFICAS.}

Perez Galaz Vicente, 1995. El Contrachapado Estructural, Ciencia e Investigación Forestal, Vol. 9,. $\mathrm{N}^{\circ} 1$, (págs 123 - 140)., Santiago, Chile.

Perez Galaz Vicente, 1996. Propiedades Físicas y Mecánicas de un Contrachapado de Coigüe Fabricado en Chile, Ciencia e Investigación Forestal, Vol. 10,. $\mathrm{N}^{\circ} 1$, (págs 5 - 32)., Santiago, Chile.

PLYWOOD ASSOCIATION OF AUSTRALIA Ltd., 1993. Design Guide for Plywood Webbed Beams, Australia.

Yagui, J. y Morales V., 1994. Estudio Teórico y Experimental de Vigas de Madera aserrada Contrachapado Sección Cajón y Doble-te. Memoria de Título de Ingeniero Civil en Obras Civiles, Dpto de Ingeniería en Obras Civiles, Universidad de Santiago de Chile.

INSTITUTO NACIONAL DE NORMALIZACION (INN), 1991. NCh 1198: MaderaConstrucciones en Madera- Cálculo. Santiago, Chile.

INSTITUTO NACIONAL DE NORMALIZACION (INN), 1989. NCh 2149: Madera AserradaDeterminación del Módulo de Elasticidad en Flexión, Santiago, Chile.

INSTITUTO NACIONAL DE NORMALIZACION (INN), 1990. NCh 1207: Pino radiataClasificación Visual para Uso Estructural- Especificación de los Grados de Calidad, Santiago, Chile. 
Perez Galaz Vicente, 1990. Manual de Cálculo de Construcciones en Madera, Instituto Forestal, Manual N 13 , Segunda Edición, Santiago, Chile.

STANDARDS ASSOCIATION OF AUSTRALIA, SAA, 1979. Timber Structures Codes, Part 1, Design Methods. AS 1720.1, Australia.

STANDARDS ASSOCIATION OF AUSTRALIA, SAA, 1979. Structural Plywood, AS 2269, Australia.

AMERICAN SOCIETY FOR TESTING AND MATERIALS, 1980. Standard Practice for Dealing with Outlying Observations, ASTM D 178 - 80, USA.

FOREST PRODUCTS LABORATORY, 1994. Wood Engineering and Construction Handbook, Prentice Hall, Madison, Wisconsin, USA. 